\title{
An unusual case of post-anaesthesia voice loss
}

\author{
Bhuwan C. Panday $\cdot$ Amitabh Dutta
}

Received: 27 June 2014 / Accepted: 26 July 2014/Published online: 20 August 2014

(C) Japanese Society of Anesthesiologists 2014

To the Editor:

A 22-year-old male suffered from fixed wide mouth opening and apparent inability to phonate clearly after bilateral osteotomy-coronoidectomy for treatment of posttraumatic chronic-progressive bilateral temporo-mandibular joint (TMJ) ankylosis with completely restricted mouth opening. Because airway access (awake fiberoptic nasal intubation), anesthetic course, and surgical TMJ mobilization were uneventful, appropriate management on the basis of diagnosis by the anesthesiologist (surgery-induced airway edema: adrenaline nebulization and intravenous steroids) and the surgeons (associated pain: multimodal analgesia) were undertaken to treat the unusual postoperative voice loss, Next morning, lack of improvement in voice profile and elimination of the possibility of other patient factors (malingering, long-term lack of awareness of adequate mouth opening) prompted surgical re-examination, which revealed bilateral TMJ dislocation. Interestingly, correction of TMJ dislocation resulted in immediate return of normal phonation.

TMJ function is crucially important to maintaining the voice quality [1]. There is evidence that TMJ disorder and/ or dysfunction (TMD) affects voice-related quality-of-life, especially vocal self-perception. Also, any chronic TMD, as in our case, induces cranio-skeletal malalignment and asymmetrical stress patterns, which further complicates the voice-loss issue [1, 2].

To conclude, for voice dysfunction after contentious TMJ surgery, the anesthesiologist must always collate-andconsider specific information on preoperative voice profile (generation, pitch) and peri-surgical factors (masseter spasm, TMJ dislocation) to arrive at a precise diagnosis, take corrective action, and avoid responses that have their own set of problems.

Conflict of interest None.

\section{References}

1. Caine A. Voice loss in performers: a pilot treatment programme to show the effect on the voice of correcting structural misalignment. Log Phon Vocal. 1998;23:32-7.

2. Pereira TC, Brassolotto AG, Conti PC, Berretin-Felix G. Temporomandibular disorders, voice and oral quality of life in women. J Appl Oral Sci. 2009;17:50-6. 